\title{
Multispectral Endoscopic Setup for Real Time Pathology Detection
}

\author{
Charly Caredda ${ }^{a, *}$, Bruno Montcel $^{a}$ and Raphaël Sablong ${ }^{a}$ \\ ${ }^{a}$ Université de Lyon, INSA-Lyon, Université Claude Bernard Lyon 1, UJM-Saint Etienne, CNRS, Inserm, \\ CREATIS UMR 5220, U1206, F69100 Lyon, France \\ *charly.caredda@creatis.insa-lyon.fr
}

\begin{abstract}
We present a multispectral endoscope using visible and near infrared illumination that can be easily adapted to flexible endoscopes. The results show that this device could be used for semi-quantitative detection of pathological tissues. () 2021 The Author(s)
\end{abstract}

\section{Introduction}

In medical imaging, endoscopy is an optical method of visualizing tissue in a cavity. Two different medical examinations are performed during endoscopic exploration: detection of pathological tissues and the characterization of pathological tissues.

For the detection of pathological tissues, most endoscopes used in clinical or preclinical studies acquire color images obtained with a continuous wave white light illumination. Recently, multispectral endoscopes, such as Narrow Band Imaging (NBI) endoscopes [1], have led to an improvement in image contrast for the detection of pathological tissues. These devices have the ability to collect tissue surface information more related to local blood volume than to its oxygenation. These devices acquire images with a large field of view and a high temporal resolution. These parameters are key factors for the detection of pathological tissues because the medical practitioners have very large cavity to explore. However these devices do not allow binary identification of tissue lesions, indeed, the identification is dependent to the visual perception of the endoscope users. Moreover, visible light only provides information about surface tissue because of the limited penetration depth of the light in this spectral range.

The second examination performed during endoscopic exploration is the pathological tissue characterization (performed once the pathological tissue has been detected). Although the reference technique consists of performing a tissue biopsy, there are characterization probes, such as hyperspectral endoscopes [2-4], that can be inserted into the endoscope's operating channel. These endoscopes acquire images with a large number of spectral bands, allowing tissue characterization. However, the field of view of the images is small $(\approx 8 \mathrm{~mm}[2])$ and the integration time for the creation of the image is long. These limitations do not allow these endosocopes to be used for both tissue detection and characterization.

Our contribution consisted in developing a prototype of a multispectral system that can be easily adapted to flexible endoscopes and more particularly to video-gastroscopes for real time detection of pathological tissues. The endoscope acquires images in the visible and near infrared range for the real time detection of surface and deep tissue lesions. Data processing are based on a simple model for the extraction of semi-quantitative parameters.

\section{Material and methods}

\subsection{Experimental Setup}

The multispectral endoscope we built is represented in Fig. 1. In this setup a rigid Karl-Storz endoscope tube (2) is used. Although the objective of this work is to improve tissue detection in flexible endoscopy, this rigid endoscope was chosen because it represents a model of choice for preclinical studies. A monochrome camera (5) (Thorlabs 340M-GE) combined with a sequential narrow band illumination with four Laser sources (8) was used to acquire a continuous video flux of multispectral images. The sequential illumination and image acquisition were performed using an input/output controller (9). The Laser sources were chosen to simplify the light injection into the endoscope (a Led illumination system will be considered in the future). Two light sources in the visible range $(447 \mathrm{~nm}$ and $532 \mathrm{~nm})$ and in the near infrared range were injected into the endoscope (793nm and $825 \mathrm{~nm}$ ). These wavelengths were chosen because of their interesting absorption characteristics by hemoglobin. Since the 
absorption of hemoglobin is 50 to 200 times greater in the visible range than in the near infrared range, different spatial information could be obtained.

A multispectral image was acquired at $8.3 \mathrm{~Hz}$ (different integration times were used for the four lifgt sources). A spectrometer (10) collected the light reflected at the injection surface of the optical fiber bundle (7). The objective was to control the stability of the light sources in order to avoid any misinterpretation of the results when analyzing the data.

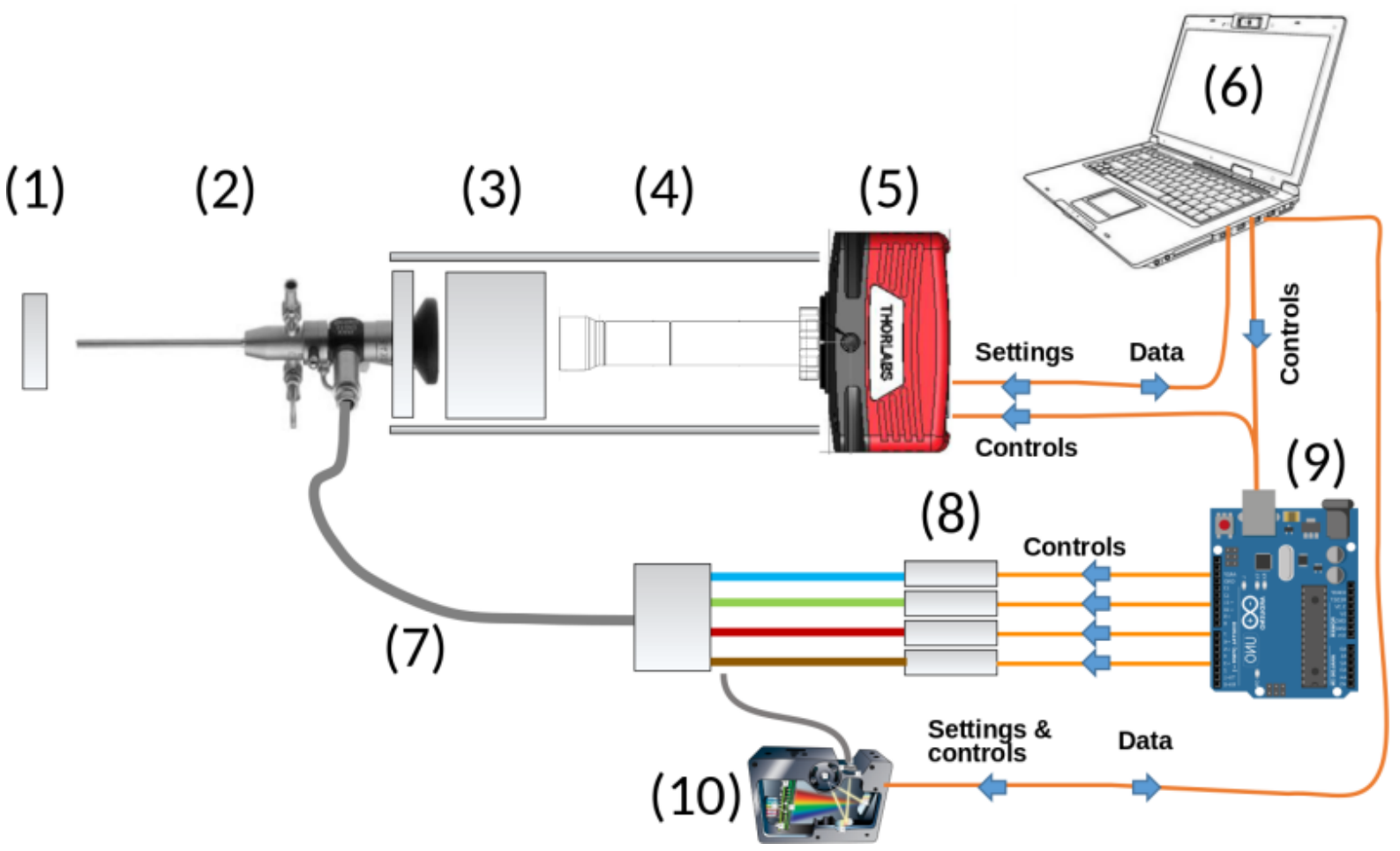

Fig. 1. Schematic of the multispectral endoscope. (1) Tissue. (2) Karl-Storz endoscope. (3-4) Lenses.

(5) Thorlabs 340M-GE camera. (6) Laptop. (7) Illumination optical fibers. (8) Multispectral sources.

(9) Input-output controller. (10) Spectrometer.

\subsection{Semi-quantitative representations}

Three semi-quantitative parameters were calculated from normalized images acquired at $447 \mathrm{~nm}\left(I_{447 \mathrm{~nm}}\right), 532 \mathrm{~nm}$ $\left(I_{532 \mathrm{~nm}}\right), 793 \mathrm{~nm}\left(I_{793 \mathrm{~nm}}\right)$ and $825 \mathrm{~nm}\left(I_{825 \mathrm{~nm}}\right)$, see Eq. $(1)$. The normalization process consists in dividing each image by a reference image obtained with a white reference target.

$$
x=\frac{I_{447 \mathrm{~nm}}}{I_{532 \mathrm{~nm}}}, \quad y=\frac{I_{825 \mathrm{~nm}}}{I_{793 \mathrm{~nm}}}, \quad z=\frac{I_{532 \mathrm{~nm}}}{I_{793 \mathrm{~nm}}}
$$

As $532 \mathrm{~nm}$ and $793 \mathrm{~nm}$ are close to isobestic points for absorbance of oxy- and deoxy-hemoglobin, the $z$ parameter can be used to get an indication of total hemoglobin concentration in tissue. Parameters $x$ and $y$ can be used to get an indication of oxygen saturation in tissue. Two different images were built in the HLS colorspace (Hue, Luminance, Saturation) to highlight the the superficial and deep contributions of photons in the tissue. The hue channel was coded by $x$ and $y$ parameters for "superficial image" and "deep image" respectively.

\section{Results and discussion}

On the left side in Fig. 2, the schematic of the multispectral endoscope is represented. On the right side, invivo images of a lip tissue are plotted. The "superficial tissue" (B) and "deep tissue" (C) images have higher contrasts than the image obtained with a white light illumination (A). Moreover, it seems that image (B) carries the information of the vascular network on the surface of the tissue and the image (C) carries the information of deeper vascular network. 


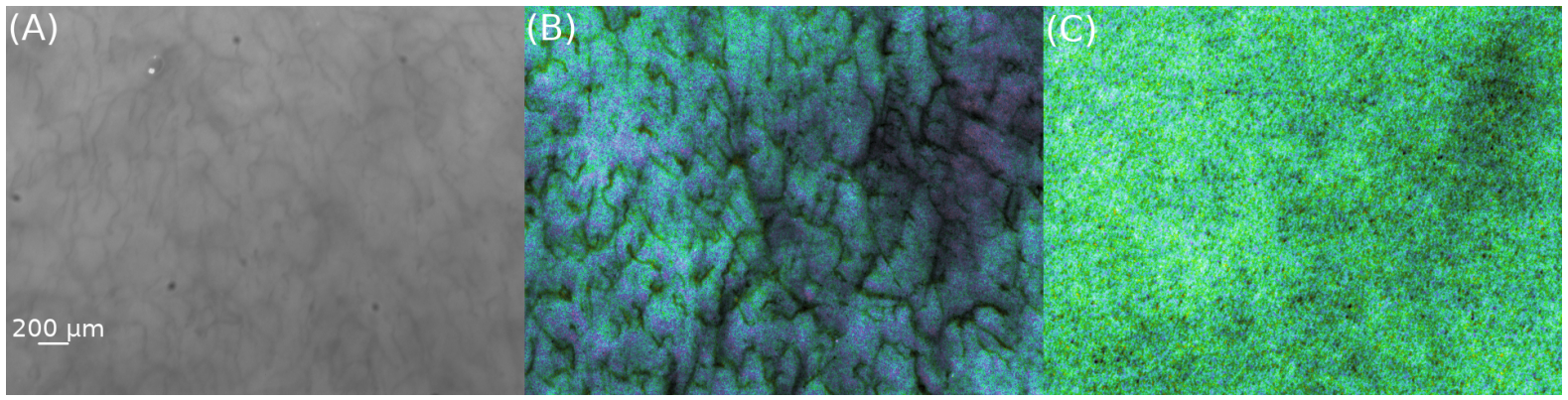

Fig. 2. Lip tissue images acquired with the multispectral endoscope. (A) Lip tissue acquired with a white light illumination. (B) "Superficial tissue" image of the lip tissue. (C) "Deep tissue" image of the lip tissue. Images (A-B-C) were obtained without the Karl-Storz endoscope (2) and the lens (3), see Fig. 1.

This multispectral endoscope could be improved. For instance, light injection and image acquisitions steps could be improved to increase the signal to noise ratio in the images. The calculated semi-quantitative parameters could be compared to simulated parameters using Monte Carlo simulations [5] in order to estimate in real time the oxygen saturation and total hemoglobin concentration in tissue allowing semi-quantitative pathological tissue detection.

\section{Conclusion}

In conclusion, we present a new mutlispectral endoscope inspired from NBI endoscopes which takes advantage of the spectral characteristics of hemoglobin in the visible and near infrared range. In future studies, the calculated semi-quantitative parameters will be compared to simulated parameters in order to estimate in real time the oxygen saturation and total hemoglobin concentration in tissue allowing semi-quantitative pathological tissue detection. This system is potentially compatible with in-vivo preclinical imaging of mouse colon.

\section{Acknowledgments}

These works were funded by LABEX PRIMES (ANR-11-LABX-0063) of Université de Lyon, within the program "Investissements d'Avenir" (ANR-11-IDEX-0007), operated by the French National Research Agency (ANR); Cancéropôle Lyon Auvergne Rhône Alpes (CLARA) within the program “OncoStarter”; Infrastructures d'Avenir en Biologie Santé (ANR-11-INBS-000), within the program "Investissements d'Avenir" operated by the French National Research Agency (ANR) and France Life Imaging (ANR-11-INBS-0006). We want to acknowledge the PILoT facility for the support provided for the image acquisition.

\section{References}

1. Kazuhiro Gono et al. Appearance of enhanced tissue features in narrow-band endoscopic imaging. Journal of biomedical optics, 9(3):568-577, 2004.

2. Erik H. Lindsley et al. The hyperspectral imaging endoscope: a new tool for in vivo cancer detection. In Dan V. Nicolau et al., editors, Imaging, Manipulation, and Analysis of Biomolecules, Cells, and Tissues II, volume 5322, pages 75 - 82. International Society for Optics and Photonics, SPIE, 2004.

3. Robert T. Kester et al. Real-time snapshot hyperspectral imaging endoscope. Journal of biomedical optics, 16(5):056005-056005, 2011.

4. Jonghee Yoon, James Joseph, Dale J. Waterhouse, A. Siri Luthman, George S. D. Gordon, Massimiliano di Pietro, Wladyslaw Januszewicz, Rebecca C. Fitzgerald, and Sarah E. Bohndiek. A clinically translatable hyperspectral endoscopy (HySE) system for imaging the gastrointestinal tract. Nature Communications, 10(1), December 2019.

5. Qianqian Fang and David A. Boas. Monte Carlo simulation of photon migration in 3d turbid media accelerated by graphics processing units. Optics express, 17(22):20178-20190, 2009. 